\title{
CONCATENATION TECHNIQUE IN CONVOLUTIONAL NEURAL NETWORKS FOR COVID-19 DETECTION BASED ON X-RAY IMAGES
}

\author{
Yakoop Razzaz Hamoud Qasim \\ Habeb Abdulkhaleq Mohammed Hassan \\ Abdulelah Abdulkhaleq Mohammed Hassan \\ Department of Mechatronics and Robotics Engineering, \\ Taiz University, Yemen
}

\begin{abstract}
In this paper we present a Convolutional Neural Network consisting of NASNet and MobileNet in parallel (concatenation) to classify three classes COVID-19, normal and pneumonia, depending on a dataset of 1083 x-ray images divided into 361 images for each class. VGG16 and RESNet152-v2 models were also prepared and trained on the same dataset to compare performance of the proposed model with their performance. After training the networks and evaluating their performance, an overall accuracy of 96.91\% for the proposed model, 92.59\% for VGG16 model and $94.14 \%$ for RESNet152. We obtained accuracy, sensitivity, specificity and precision of $99.69 \%, 99.07 \%, 100 \%$ and $100 \%$ respectively for the proposed model related to the COVID-19 class. These results were better than the results of other models. The conclusion, neural networks are built from models in parallel are most effective when the data available for training are small and the features of different classes are similar.
\end{abstract}

\section{KEYWORDS}

Deep Learning, Concatenation Technique, Convolutional Neural Networks, COVID-19, Transfer Learning.

\section{INTRODUCTION}

In late 2019, a new strain of coronavirus emerged and was named coronavirus disease 2019 (COVID-19), and the first case of COVID-19 infection was registered in Wuhan, China [1], there are many symptoms that appear on a person with COVID-19 such as fever, cough, cold ,shortness ordifficulty in breathing, problems in respiratory systems and pain in the joints[2]. According to The World Health Organization(WHO) the number of deaths by COVID-19 has exceeded 506 thousand, the number of confirmed cases has reached over 10 million and the number of recovery cases has reached 5.24 million[3]. Given the rapid spread of COVID-19 and its devastating effects on the lifestyle of people and their lives, countries resorted to applying the general quarantine to stop the spread of COVID-19, which led to catastrophic consequences for countries economy, so it was necessary to develop means to detect COVID-19, because early and wide detection means reducing the spread of the disease. According to WHO, the respiratory tract infection, specifically the lung infection is considered one of signs and symptoms of COVID19[3]. 
As is well known, a radiological diagnosis X-Ray and CT images can be used to detect and diagnose respiratory problems, and using radiological diagnosis, this helps to overcome the shortage and scarcity of the examination tools and allowing to examine the largest possible population for the availability of radiological diagnostic devices in most hospitals and laboratories. But there is a disadvantage in radiological diagnosis, due to the necessity of needing an expert in radiology to confirm and diagnose the disease, which leads again to slowing the process of diagnosis and increase the cost, so the approach suggested in this paper is to use deep Convolutional Neural Network (CNN) to diagnose and detect COVID-19.The model we proposed is a CNN model which is based on the concatenation of NASNet-Mobile [4] and MobileNet [5] for classified three classes COVID-19, normal and pneumonia.

\section{RELATED WORK}

To diagnose COVID-19 disease by using convolutional neural network CNN based on X-ray images, several searches have been introduced in this field.In [6] the authors used a network consisting of Xception [7] and ResNet50-v2 [8] in parallel on a dataset of 15085X-ray images. They used a cross validation strategy to training the network, this proposed model achieved an average overall accuracy of $94.4 \%$. In [9] the authors presented a CNN model named nCOVNet which is based on the VGG19 model [10], transfer learning was used to retrain the model on a dataset consisting of 284 X-ray images for the two classes COVID-19 and normal. After training the model achieved an overall accuracy of $88.10 \%$, sensitivity of $97.62 \%$ and specificity of 78.57\%. In [11] the authors presented a CNN model called CoroNet based on Xception architecture. The model was trained on a dataset consisting of $1251 \mathrm{X}$-ray images for four classes COVID-19, normal, bacterial pneumonia and viral pneumonia. The model achieved an overall accuracy of $89.69 \%$, specificity of $93 \%$ and $98.2 \%$ related to the COVID-19 class. The model was also trained in three classes COVID-19, normal and pneumonia (mixture of viral and bacterial), and obtained an overall accuracy of 95\%. In [12] the authors used transfer learning technique to train the VGG19model on a dataset of 445 X-ray images for COVID-19 and normal classes. They achieved an overall accuracy of $96.3 \%$, sensitivity of $97 \%$ and precision of $91.7 \%$ related to the COVID-19 class. In [13] transfer learning technology was used to train VGG19 [10], MobileNet-v2 [14], Inception [15], Xception [7] and Inception ResNet-v2 [16] on a dataset consisting of 1428 X-ray images for three classes COVID-19, normal and bacterial pneumonia, They got $99.10 \%$ sensitivity for COVID-19 class from MobileNet model. After that, a group of $\mathrm{X}$-ray images of viral pneumonia was added to the previous dataset and then trained on MobileNet model, the model achieved an overall accuracy of $94.72 \%$ and $96.78 \%$ accuracy for the COVID-19 class.

The remainder of this paper can be summarized as follows. In section three we explain the methodology which consists of the proposed model and the dataset which is used for training the model, then in section four we will show the results we obtained from the proposed model and other models, then in section five we will discuss the results and in section six we will show the conclusion that we reached.

\section{MeTHOdOLOGY}

\subsection{Dataset}

Due to the lack of available resources for chest X-ray images of those people with COVID-19, the dataset that is used in this paper were collected from several sources. The first source which is COVID-19 image data collection [17], is available on GitHub website and only 142 images were taken for COVID-19 class. The second source which is COVID-19 Radiography database[18], is 
available on Kaggle website, contains 219 cases for COVID-19, 1341 normal and 1345 for viral pneumonia, all images from COVID-19 cases were taken as well as 361 from normal cases. The third source which is chest $\mathrm{x}$-ray images (pneumonia) [19], is available on Kaggle website, contains 5863 x-ray images for two classes normal and pneumonia(a mixture of viral and bacterial),and 361 cases were taken for the pneumonia class. After collecting the images from the three sources, we had 1083 images divided into 316 images for each class. We divided the dataset into 759 images for training and 324 for validation. We did not perform any process to check the accuracy of the data, we satisfied with the reliability of the sources and the data was divided randomly.

\subsection{Convolutional Neural Network}

Convolutional neural network $(\mathrm{CNN})$ is type of neural network which is very effective in the field of image classification and problem solving of image processing. The word convolution refers to the filtering process that happen in this type of networks [20]. This networks consist of multiple layers which are: The convolution layer which is the core layer and it works by placing a filter over an array of image pixels, this then creates what is known as a Convolved Feature Map (CFM). The pooling layer which reduces the sample size of feature map, this makes processing too faster [20], by reducing the parameters that the network needs to process. A Rectified Linear Unit Layer (Relu) that acts as an activation function ensuring Non-Linearity. A Fully Connected Layer allowing us to perform classification on our dataset.

COVID-19 symptoms are often identical to the symptoms of other viral pneumonia and because of the similarity of the effect of COVID-19 and the effect of the other infections on the lung [21, 22]. It is difficult to diagnose with $X$-Ray images except by an experienced $X$-Ray expert. But is easier to diagnose with the neural networks. With the great similarity between the effect of COVID-19 and other pneumonia, such as viral pneumonia, it is necessary to build a deeper neural network in order to be able to classify properly, but this type of network requires a large dataset and high computing capabilities for training. Whereas building a network of two models in parallel has the ability to learn different and overlapping high-level and low-level features [23].

So we built a network of two models in parallel (concatenation) to have a high ability to extract and classify features properly, so we used NASNet-Mobile [4] and MobileNet [5] to configure this network. NASNet-Mobile was chosen for several reasons, including the small number of parameters as well as the ability to achieve state-of-the-art result and less complexity [4, 24]. MobileNet was also chosen for several reasons, including the small size and lack of complexity in it's structure because it is based on the DepthWise Separable Convolution [5, 25].As noted in figure.1, global average pooling, global max pooling and flatten have been linked in parallel to improve the network performance and help to prevent overfitting and create a feature map for each category in the last layers [26]. To compare the performance of the proposed network, two models VGG16 [27] and RESNET152-v2 [28] were prepared and trained on the same dataset. These two models are the most popular used. The VGG16 model is very popular and widely used because of pre-trained weights were made freely available online. RESNET152-v2 also is one of the most popular models which "introduced the concept of Residual Learning in which the subtraction of features is learned from the input layer by using shortcut connection"[29]. 


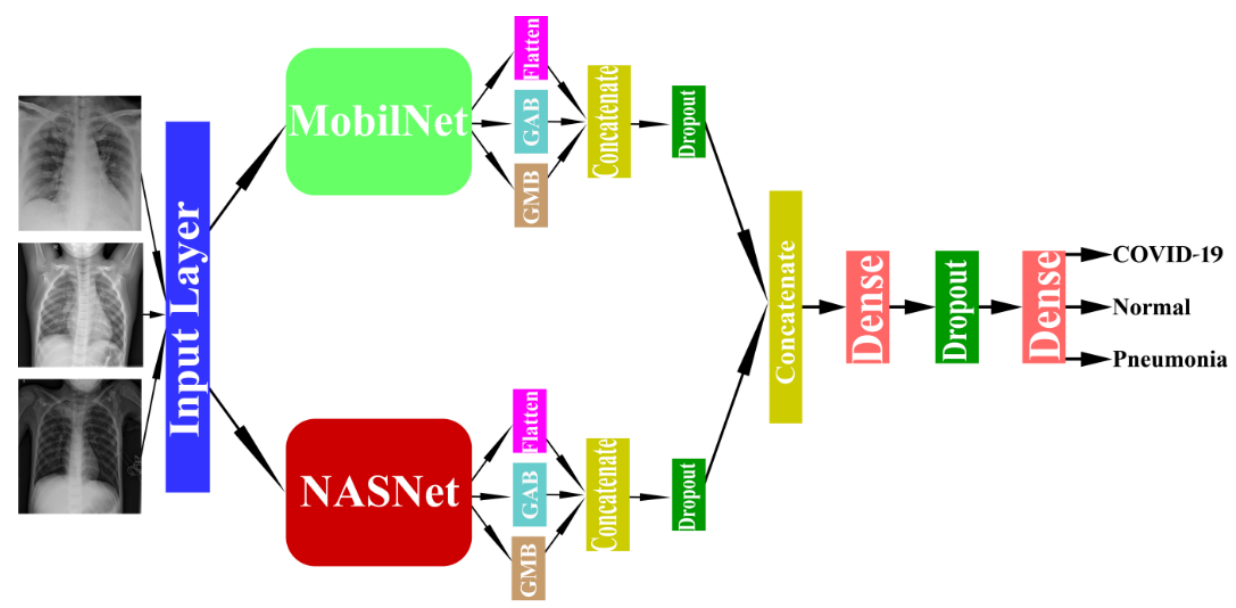

Input Layer $\square$ MobileNet $\square$ NASNet $\square$ Global Average Pooling $\square$ Global Max Pooling

Flatten Layer Concatenate Layer $\square$ Dropout Layer $\square$ Dense Layer

Figure. 1. The architecture of the proposed network, concatenation of two models

\subsection{Transfer Learning}

A technique for reusing the weights of pre-trained network on a task similar to the current task as classification. We have used this technique to train all previously mentioned models, which makes it easier for us to train new models in less time and few computing resource.

Table 1 Training Hyper-Parameters.

\begin{tabular}{|c|c|c|c|}
\hline \multirow{2}{*}{$\begin{array}{c}\text { Hyper- } \\
\text { Parameters }\end{array}$} & \multicolumn{3}{|c|}{ Models } \\
\cline { 2 - 4 } Batch Size & VGG16 & ResNet152 & Proposed \\
\hline Learning Rate & $1 \mathrm{e}-3$ & 32 & 32 \\
\hline Epochs & 50 & $1 \mathrm{e}-3$ & $1 \mathrm{e}-3$ \\
\hline Image Size & 200,200 & 200,200 & 200,200 \\
\hline $\begin{array}{c}\text { Optimizer } \\
\text { Function }\end{array}$ & Adam & Adam & SGD \\
\hline $\begin{array}{c}\text { Data } \\
\text { Augmentation }\end{array}$ & No & No & No \\
\hline Loss Function & $\begin{array}{c}\text { Categorical- } \\
\text { crossentropy }\end{array}$ & $\begin{array}{c}\text { Categorical- } \\
\text { crossentropy }\end{array}$ & $\begin{array}{c}\text { Categorical- } \\
\text { crossentropy }\end{array}$ \\
\hline $\begin{array}{c}\text { Validation } \\
\text { Split }\end{array}$ & 0.30 & 0.30 & 0.30 \\
\hline
\end{tabular}

\section{RESUlts}

After the training the models and evaluating their performance with confusion matrix we obtained the following results. 


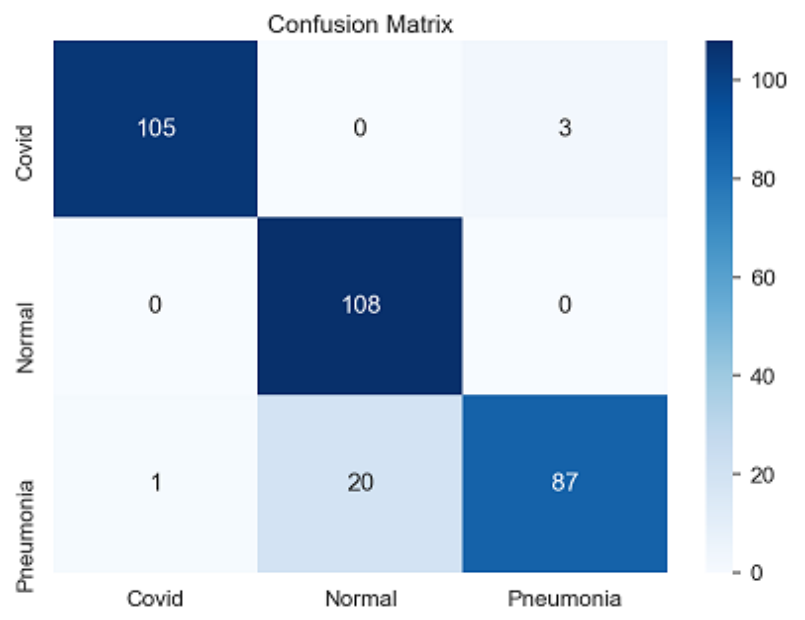

Figure.2. Confusion Matrix for the VGG16 Model

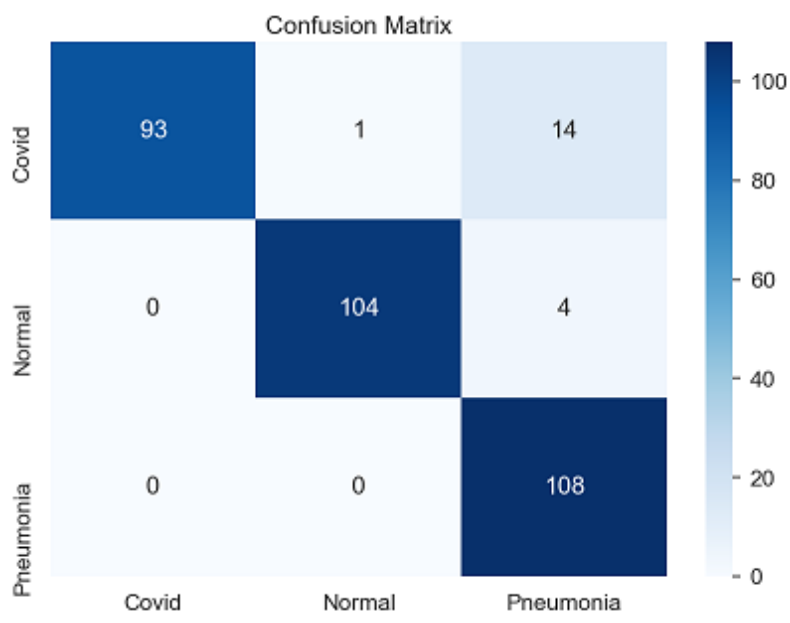

Figure.3. Confusion Matrix for the ResNet152 Model

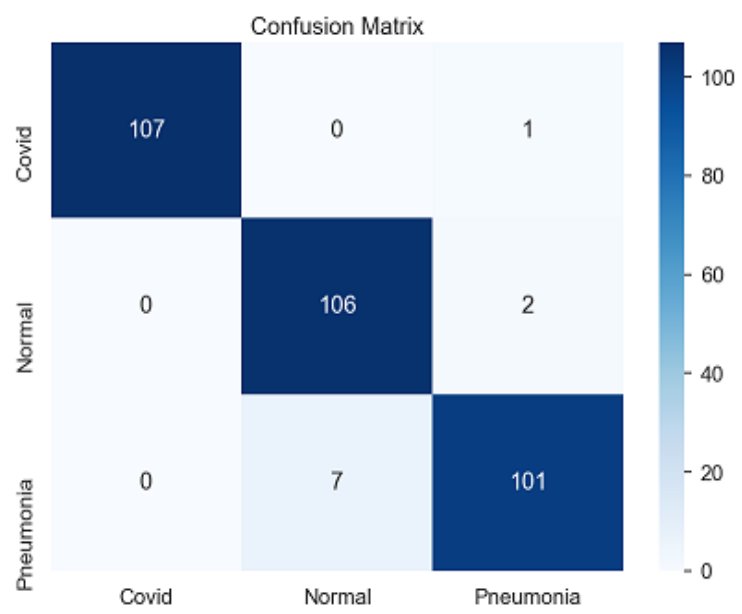

Figure. 4. Confusion Matrix for the Proposed Model 
Confusion Matrix is a matrix that is used for describing the performance of the classification model on a set of validation data whose true values are already known, confusion matrix is useful for measuring accuracy, sensitivity, specificity, precision and F-Measure. To calculate these values it is necessary to know four parameters which are True Positive (TP), True Negative(TN),False Positive (FP) and False Negative(FN) and how to compute them, Suppose we wanted to calculate the four parameters mentioned previously for one of the classes and let's assume that the class isCOVID-19. So we have as follows.

TP: refers to the cases that belong to the COVID-19 cases and were classified under COVID-19 cases. TN: refers to the cases that belong to the other cases normal and pneumonia, and were not classified under COVID-19 cases, FP: refers to the cases that belong to the other cases and were classified under COVID-19 cases and FN: refers to the cases that belong to COVID-19 cases and were not classified under COVID-19 cases. Tables 2, 3 and 4 shows the results of the four parameters for the three models related toCOVID-19, normal and pneumonia classes respectively. Now based on these parameters we can calculate the following evaluation metrics which are accuracy for each class, sensitivity, specificity, precision and F-measure.

Sensitivity for COVID-19 tell us what percentage of cases with COVID-19 were correctly identified, Specificity for COVID-19 tell us what percentage of cases without COVID-19 were correctly identified, Precision tell us what percentage of cases that actually belong to the COVID19 cases from all cases that classified as COVID-19. F-Measure which is the harmonic mean of sensitivity and precision.

Overall Accuracy $=$ correct predictions $/$ total predictions.

Accuracy for each class $=(\mathrm{TP}+\mathrm{TN}) /(\mathrm{TP}+\mathrm{FP}+\mathrm{TN}+\mathrm{FN})$

Sensitivity $=\mathrm{TP} /(\mathrm{TP}+\mathrm{FN})$.

Specificity $=\mathrm{TN} /(\mathrm{TN}+\mathrm{FP})$.

Precision $=\mathrm{TP} /(\mathrm{TP}+\mathrm{FP})$

F-Measure $=2 *$ Sensitivity $*$ Precision $/$ (precision + Sensitivity)

From the previous Confusion Matrixes, we calculated the four parameters and presented them in the following tables for each class.

Table 2 Four parameters related to the COVID-19 class.

\begin{tabular}{|c|c|c|c|c|}
\hline Model & TP & FP & TN & FN \\
\hline VGG16 & 105 & 1 & 215 & 3 \\
\hline RESNet152 & 93 & 0 & 216 & 15 \\
\hline Proposed & 107 & 0 & 216 & 1 \\
\hline
\end{tabular}

Table 3 Four parameters related to the Normal class.

\begin{tabular}{|c|c|c|c|c|}
\hline Model & TP & FP & TN & FN \\
\hline VGG16 & 108 & 20 & 196 & 0 \\
\hline RESNet152 & 104 & 1 & 215 & 4 \\
\hline Proposed & 106 & 7 & 209 & 2 \\
\hline
\end{tabular}

Table 4 Four parameters related to the Pneumonia class.

\begin{tabular}{|c|c|c|c|c|}
\hline Model & TP & FP & TN & FN \\
\hline VGG16 & 87 & 3 & 213 & 21 \\
\hline RESNet152 & 108 & 18 & 198 & 0 \\
\hline Proposed & 101 & 3 & 213 & 7 \\
\hline
\end{tabular}


From parameters value, we calculated overall accuracy and accuracy, sensitivity, Specificity, precision and F1-score for each class and presented the results in the following table.

Table 5 Overall accuracy and Evaluation Metrics for each class from three models.

\begin{tabular}{|l|l|l|l|}
\hline \multirow{2}{*}{\multicolumn{1}{|c|}{ Evaluation Metrics }} & \multicolumn{3}{c|}{ Models } \\
\cline { 2 - 4 } & VGG16 & RESNet152 & Proposed \\
\hline Overall Accuracy & 92.59 & 94.14 & 96.91 \\
\hline COVID19 Accuracy & 98.77 & 95.37 & 99.69 \\
\hline Normal Accuracy & 93.83 & 98.46 & 97.22 \\
\hline Pneumonia Accuracy & 92.59 & 94.44 & 96.91 \\
\hline COVID19 Sensitivity & 97.22 & 86.11 & 99.07 \\
\hline Normal Sensitivity & 100 & 96.3 & 98.15 \\
\hline Pneumonia Sensitivity & 80.56 & 100 & 93.52 \\
\hline COVID19 Specificity & 99.53 & 100 & 100 \\
\hline Normal Specificity & 90.74 & 99.54 & 96.76 \\
\hline Pneumonia Specificity & 98.61 & 91.67 & 98.61 \\
\hline COVID19 Precision & 99.06 & 100 & 100 \\
\hline Normal Precision & 84.38 & 99.05 & 93.81 \\
\hline Pneumonia Precision & 96.67 & 85.71 & 97.12 \\
\hline COVID19 F-Measure & 98.13 & 92.54 & 99.53 \\
\hline Normal F-Measure & 91.53 & 97.66 & 96.93 \\
\hline Pneumonia F-Measure & 87.88 & 92.31 & 95.29 \\
\hline
\end{tabular}

\section{DisCUSSION}

Since the goal of this paper is to detect and diagnose COVID-19, we will focus on the overall accuracy of the models and the results related to the COVID-19 class, which are accuracy, sensitivity, specificity, precision and F-measure. From table 5, we note that the VGG16 model has achieved $92.59 \%$ overall accuracy, the RESNet 152 model has achieved $94.14 \%$ overall accuracy and the proposed model has achieved $96.91 \%$ overall accuracy which is the best. Also from table 5, we note that the VGG16 model has achieved 98.77\% accuracy, 97.22\% sensitivity, 99.53\% specificity, $99.06 \%$ precision and 98.13 F-measure for the COVID-19 class. And the ResNet152 model has achieved $95.37 \%$ accuracy, $86.11 \%$ sensitivity, $100 \%$ specificity, $100 \%$ precision and 92.54 F-measure for COVID-19 class. It is noticeable that the VGG16 andRESNet152 models have achieved a good performance, but the VGG16 model has outperformed the RESNet152 through accuracy, sensitivity and F-measure, but achieved fewer results through specificity and precision. By reviewing the results of the proposed model in table 5 , we note that the model has outperformed the previous two models through the results of the COVID-19 class while having some deficiencies in other classes. We note that the sensitivity of the proposed model is very high, which means that the model is very sensitive to images of COVID-19 class, and very suitable for detect COVID-19. When a large dataset of COVID-19 is available, we will increase the number of paths and test the model on it.

\section{CONClusion AND Future Work}

we have provided a deep neural network consisting of two models in parallel with low parameters and does not need a large dataset or highly computing resources for training. This network has the ability to extract features well, learn and classify them with high accuracy. Then we compared the network performance with two networks that are popular which are VGG16 and RESNet152- 
v2 in terms overall accuracy, accuracy for each class, sensitivity, specificity and F-measure. The result of this network were very good and satisfactory compared to the result obtained from other networks. Thus we come to conclusion that the neural networks in this way are very effective in the event that the available dataset are few, and there is a great similarity in the features between the classes. In the future works, we will test the model on a different dataset and develop the model so that it is very effective in medical diagnostics.

\section{REFERENCES}

[1] N. Zhu, D. Zhang, W. Wang, X. Li, B. Yang, J. Song, X. Zhao, B. Huang, W. Shi, R. Lu, P. Niu, F. Zhan, X. Ma, D. Wang, W. Xu, G. Wu, G.F. Gao, W. Tan, A novel coronavirus from patients with pneumonia in China, 2019 N. Engl. J. Med., 382 (2020), pp. 727-733

[2] C. Huang, Y. Wang, X. Li, L. Ren, J. Zhao, Y. Hu, L. Zhang, G. Fan, J. Xu, X. Gu, Z. Cheng, T. Yu, J. Xia, Y. Wei, W. Wu, X. Xie, W. Yin, H. Li, M. Liu, Y. Xiao, H. Gao, L. Guo, J. Xie, G. Wang, R. Jiang, Z. Gao, Q. Jin, J. Wang, B. Cao, Clinical features of patients infected with 2019 novel coronavirus in Wuhan, China Lancet, 395 (2020), pp. 497-506

[3] WHO Coronavirus disease. Last Accessed : 30 Jun 2020

[4] Barret Zoph, Vijay Vasudevan, Jonathon Shlens, Quoc V. Le. Learning Transferable Architectures for Scalable Image Recognition. arXiv:1707.07012v4. 2018.

[5] Andrew G. Howard, Menglong Zhu, Bo Chen, Dmitry Kalenichenko, Weijun Wang, Tobias Weyand, Marco Andreetto, Hartwing Adam. MobileNets: Efficient Convolutional Neural Networks for Mobile Vision Application. arXiv:1704.04861v1. 2017.

[6] Mohammad Rahimzadeh, Abolfal Attar. A modified deep convolutional neural network for detecting COVID-19 and pneumonia from X-ray images based on the concatenation of Xception and ResNet50v2. (2020) 2020.100360.

[7] Chollet F. Xception: deep learning with depthwise separable convolution. In: proceedings pf the IEEE conference on computer vision and pattern recognition. 2017. P. 1251-8.

[8] He K, Zhang X, Ren S, Sun J. Identity mappings in deep residual networks. In:European conference on computer vision. Springer, 2016. p. 630-45.

[9] Harsh Panwar, P.K. Gupta, Mhammad Khubeb Siddiqui, Ruben Morales-Menendez, Vaishnavi Singh. Application of deep learning for fast detection of COVID-19 in X-ray using nCOVnet. (2020) 2020.109944.

[10] Simonyan K, Zisserman A (2014) Very deep convolutional networks for large-scale image recognition. arXiv, 1409: p. 1556.

[11] Asif Iqbal Khan, Junaid Latief Shah, Mohammad Mudasir Bhat. CoroNet: A deep neural network for detection and diagnosis of COVID-19 from chest x-ray images. (2020) 2020.105581.

[12] Shahank Vaid, Reza Kalantar, Mohit Bhandari. Deep learning COVID-19 detection bias: accuracy through artificial intelligence. 2020. https://doi.org/10.1007/s00264-020-04609-7.

[13] Ioannis D. Apostolopoulos, Tzani A. Mpesiana. Covid-19: Automatic detection from X-ray images utilizing transfer learning with convolutional neural networks. 2020. https://doi.org/10.1007/s13246020-00865-4.

[14] Howard AG Zhu M Chen B et al (2017)MobileNets: efficient convolutional neural networks for mobile vision applications. arXiv preprint arXiv: 170404861.

[15] Szegedy C, Liu W, Jia Y, Sermanet P, Reed S, Anguelov D, et al. Going deeper with convolutions.Boston, MA, 2015,: IEEE Conference on Computer Vision and Pattern Recognition (CVPR); 2015. P. 1-9.

[16] Szegedy C, Ioffe S, Vanhoucke V, Alemi A. Inception-v4, inception-resnet and the impact of residual connections on learning. 2016.

[17] Cohen JP. COVID-19 image data collection. (2020) https://github.com/ieee8023/covid-chestxraydataset.

[18] Tawsifur Rahman, M.E.H. Chowdhury, A. Khandakar. COVID-19 Radiography database. https://www.kaggle.com/tawsifurrahman/covid19-radiography-database .

[19] Paul Mooney. Chest X-ray Images (Pneumonia). https://www.kaggle.com/paultimothymooney/chestxray-pneumonia . 
[20] Sumit Saha. A Comprehensive Guide to Convolutional Neural Network -the ELI5 way. https://towordsdatascience.com/a-comprehensive-guide-to-convolutional-neural-network-the-eli5way-3bd2b1164a5 . Last accessed on Jun 2020.

[21] Zawn Villines. What is the relationship between pneumonia and COVID-19?. https://www.medicalnewstoday.com/articles/pneumonia-and-covid-19\#summary . last accessed on Jun 2020.

[22] Jill Seladi-Schulman. What to Know About COVID-19 and Pneumonia. https://www.healthline.com/health/coronavirus-pneumonia. Last accessed on Jun 2020.

[23] Sabyasachi Sahoo. Grouped Convolutiona - convolutions in parallel. https://towordsdatascience.com/grouped-convolutions-convolutions-in-parallel-3b8cc847e851 . Last accessed on May 2020.

[24] Sik-Ho Tsang. Review: NASNet-Neural Architecture Search Network (Image Classification). https://medium.com/@sh.tsang/review-nasnet-neural-architecture-search-network-imageclassification-23139ea0425d . Last accessed on APRIL 2020.

[25] Sik-Ho Tsang. Review:MobileNetv1-Depthwise Separable Convolution (Light Weight Model). https://towardsdatascience.com/review-mobilenetv1-depthwise-separable-convolution-light-weightmodel-a382df364b69. Last accessed MAY 2020.

[26] Chris. What are Max Pooling, Average Pooling, Global Max Pooling and Global Average Pooling. https://www.machinecurve.com/index.php/2020/01/30/what-are-max-pooling-average-poolingglobal-max-pooling-and-global-average-pooling/ . Last accessed on MARCH 2020.

[27] Karen Simonyan, Andrew Zisserman. Very Deep Convolutional Networks for Large-Scale Image Reconition. arXiv:1409.1556. 2014.

[28] Kaiming He, Xiangyu Zhang, Shaoqing Ren, Jian Sun. Deep Residual Learning for Image Recognition. arXiv:1512.03385. 2015.

[29] Han, Seung \& Park, Gyeong \& Lim, Woohyung \& Kim, Myoung \& Na, Jung-Im \& Park, Ilwoo \& Chang, Sung. (2018). Deep neural networks show an equivalent and often superior performance to dermatologists in onychomycosis diagnosis: Automatic construction of onychomycosis datasets by region-based convolutional deep neural network. PLOS ONE. 13. e0191493. 10.1371/journal.pone.0191493.

\section{AUTHORS}

Yakoop Qasim A student in the fourth level, department of Mechatronics and Robotics Engineering, Al-Saeed college of Engineering and Information Technology at Taiz University. Professional in designing control systems, modeling and simulation of dynamic systems. Intelligent system programmer and interested in the field of artificial intelligence.

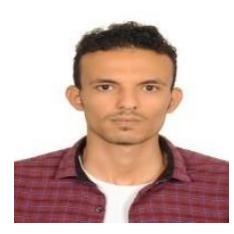

Habeb Hassan A student in the fourth level, department of Mechatronics and Robotics Engineering, Al-Saeed college of Engineering and Information Technology at Taiz University. Have many skills in programming and designing, hope in the future to prepare master's degree in artificial intelligence and how to use it in robotics.

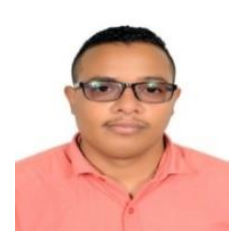

Abdulelah Hassan A student in the third level, department of Mechatronics and Robotics Engineering, Al-Saeed college of Engineering and Information Technology at Taiz University.

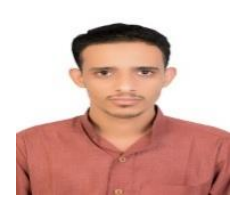

(C) 2020 By AIRCC Publishing Corporation. This article is published under the Creative Commons Attribution (CC BY) license. 